\title{
La lectura: un problema para la sociedad de la información
}

\author{
Elsa Margarita Ramírez Leyva \\ Centro Universitario de Investigaciones Bibliotecológicas \\ de la UNAM, 04510, México D.F., Tel: 56-23-03-29 \\ E-Mail: eramirez@ servidor.unam.mx
}

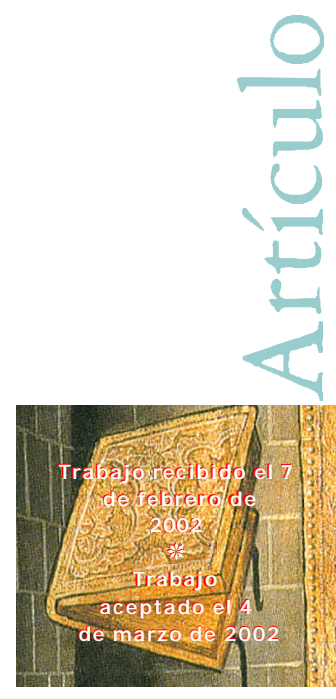

\author{
Para Gloia Escamilla González (q.epd) \\ La soiedadmopuedeser tal sinla commicaión \\ ynopuredetransfomarsesinlainformaión \\ J. Antonio Paoli \\ La letura, antes queuna destreza, esunplacer \\ quemosedesabrepor azar
}

Equipo Peonza

\section{RESUMEN}

Este artículo abre la reflexión sobreel problema que representala lectura para la sociedad de la información, la cual deberá distinguirse por contar con ciudadanos capaces de acceder, usar la información y convertirla en un bien para el beneficio individual y colectivo que conduzca al progreso. Sin embargo el estudio de la OECD, dado a conocer recientemente, sobre la capacidad para comprender textos, evaluar informaciones, construir hipótesis y aprovechar conocimientos, reveló serios problemas en la capacidad lectora en jóvenes que concluyeron la educación media en los 36 países encuestados.

Por otra parte el desempeño de las instituciones educativas, en particular la educación superior, de capacitar a los alumnos para desarrollar habilidades queles permitan acceder, usar la información y transformarla en conocimiento también ha mostrado insuficiencias.

Al respecto, la American Library Association definió seis habilidades básicas que todo estudiante debe adquirir, supuestamente para dominar las habilidades de lectura. El estudio de la OECD obligará a revisar los factores que afectan y deterioran la capacidad lectora; y de paso el gusto por ella.

Palabras clave: Lectura, Conducta Lectora, Habilidades Informativas, Sociedad de la Información. 


\title{
WRITING: A PROBLEM FOR THE INFORMATION SOCIETY ELSA MARgARITA RAMÍREZ-LEYVA
}

\begin{abstract}
A reflection on the problem of writing for the information society which should count with citizens capable of using information for the individual and collective benefit leading to progress. However, a recent OECD's survey on the ability to understand texts, evaluate information, build hypothesis and profit from knowledge, revealed serious problems in the 36 countries involved in the testing of writing abilities of youth that had just concluded middle education. But also the performance in the higher education systems revealed certain difficulties in the capabilities for accessing, using and transforming information. Related to this, the American Library Association defined six basic capabilities that all students should acquire in order to be able to master the writing abilities. The OECD survey will lead to a review of factors that affect and deteriorate the joy of reading.

Key Words: Reading, Reading Behavior, Information Ability, Information Society.
\end{abstract}

\section{LA CULTURA DE LA INFORMACIÓN DEL SIGLO XXI}

lo largo de la historia de la humanidad, la comunicación y la información han sido elementos constitutivos de la sociedad. ${ }^{1}$ La comunicación se produce eomo resultado de la información, el acto de comunicación (emisión y recepción) y de la comprensión, en y por, la vida en sociedad. A su vez en la comunicación se gesta el interés y la necesidad de desarrollarse y conservar los múltiples datos culturales producidos en las distintas épocas y regiones; es decir queel proceso de civilización, y por tanto la cultura, competen a la comunicación, son causa y efecto de la misma. Por su parte, los individuos se adaptan a los cambios que produce la información mediante la comunicación. Se puede considerar entonces como principio que el mundo de las posibilidades sociales está circunscrito a las posibilidades decomunicación y de desarrollo cultural, tanto a nivel individual como social.

Por tanto la escritura y la lectura se han constituido en parámetros que miden el desarrollo cultural y económico de un pueblo.

Es en especial el siglo XX el que se distingue por emprender acciones mundiales encaminadas a erradicar el analfabetismo y superar los niveles de educación básica. Más aún, se considera a la educación como un derecho universal consolidado a lo largo de cien años, por lo que era esperable que el siglo XXI fuera recibido por pueblos

1 Niklas Luhmann y Raffaele deG eorgi. Texáadelasoiecad México:U.deG ., UIA, ITSO , 1993, p. 15. 
letrados y preparados para vivir en la sociedad de la información. Sin embargo la lectura, tanto como capacidad y como práctica, está en crisis y de ello se culpa a la escuela y a la televisión, y también se empieza a involucrar en esto a Internet.

Es un hecho que estamos ingresando a la sociedad de la información sin lectores, ello no significa que éstos sean analfabetas, sino más bien que se ha dado una notabledisminución en las aptitudes y el gusto por leer. Lo cual resulta paradójico parala cultura actual, que requiere individuos altamente capacitados para acceder, usar y comunicar información.

La sociedad actual denominada "dela información o del conocimiento" debería te ner pueblos informados, y capacitados para informar a través de diferentes medios y para utilizar la información en beneficio no sólo personal sino también social. Actualmente somos testigos de cambios importantes en la cultura de la información, principalmente debido a la presencia de la computadora, de la hipertextualidad digital y de Internet que han empezado a competir con los medios impresos. D e la misma forma que en otro momento las tecnologías modificaron y hasta revolucionaron la cultura de lainformación delas sociedades orales y manuscritas. Ahora, como en épocas anteriores, se crearon, en función de sus medios, formas de informar y de informarse; es decir, modos de recordar, transcribir y transmitir el producto del conocimiento de sí mismo y de su entorno; de sus descubrimientos y de sus inventos.

A la civilización, como fase última de la evolución social (la preceden el salvajismo y la barbarie) se la identifica por lo general con el surgimiento del lenguaje escrito; el cual marca el pasaje del pensamiento simbólico al lógico-abstracto. Por medio de éste se ha plasmado y transmitido la cultura y sus esquemas de pensamiento: lenguaje, creencias, mitos, sentimientos, valores conformados por las experiencias que comparten, comprenden de manera ritual y enseñan (enculturando a los nuevos miembros) los grupos sociales. Hoy la tecnología de la información y la comunicación (TIC) creala expectativa de alcanzar niveles superiores de desarrollo a los logrados hasta el momento, al extender, a través de los nuevos medios, concretamente Internet, lainformación para todos. ${ }^{2}$ Se podría decir entonces quelaforma deenculturar a los integrantes de la sociedad contemporánea será a través de la TIC.

La cultura se crea y desarrolla en la medida en que un grupo social resuelve los problemas planteados por las necesidades de cohesión, progreso, identidad, estabilidad y diferenciación entre grupos y en tanto defiende su cultura para mantener la interacción social y asegurar su preservación y evolución, ya sea en sistemas sociales cerrados (profesionistas de un campo, grupos religiosos, tribus, familia, etcétera), o abiertos (grupos provenientes de diferentes sectores: universidad, empresa).

2 El Plan INFO XXI: La Sociedad de la Información para todos. El Plan establece principios y acciones dirigidas a los gobiernos con el fin de crear las condiciones para que todo ciudadano acceda a la información. Cf López Y epes, José. Lapdíticadela sociedadenEspaña D ocumento presentado en: Jornadas de documentación administrativay sociedad de la información. Zaragoza, 22-24 de octubre de 2001, p. 10. 
La comunicación necesita de medios y formas como el lenguaje, transmitido por diferentes canales, para asegurar la comunicación y la información entre los miembros del grupo y de éste con el exterior. Por lo mismo existe un lenguaje para comunicarse con el grupo y otro para comunicarse con el exterior.

D e acuerdo con la propuesta de Schein ${ }^{3}$ los elementos básicos de la cultura se dividen en tres categorías: a) artefactos y creaciones, en los que se ubican la tecnología, el arte y los esquemas de conducta visibles y audibles; b) los valores, y entre los cuales se encuentran los que pueden ser confrontables con la naturaleza y aquellos que pueden ser confrontables sólo por consenso social; y c) las presunciones básicas, en donde se encuentran la relación con el entorno, con la realidad, el tiempo y el espacio, y con la naturaleza del género humano. Estos elementos básicos resultan de las experiencias que obtiene un grupo al solucionar sus propios problemas, tener experiencias comunes y formar una visión compartida de ellos a la vez que del mundo con el que interactúan, así como de sus símbolos culturales. ${ }^{4}$ D e tal forma la cultura se va aprendiendo mediante la interacción entre los integrantes del grupo, de manera que una parte se transformay otra se preserva. Concretamente, los elementos que ledan identidad al grupo son el lenguaje, los mitos, los rituales, los símbolos, el arte.

Estas ideas de Schein las encontramos sintetizadas en la definición de cultura propuesta por A. Aguirre:

[...]sistema de conocimientos que nos proporciona un modelo de realidad, a través del cual damos sentido a nuestro comportamiento. Este sistema está formado por un conjunto de elementos interactivos fundamentales, generados y compartidos por el grupo al cual identifican por lo que son transmitidos a los miembros (enculturación), siendo eficaces en la resolución de los problemas;[... ${ }^{5}$

La que nos sirve como base para entender la cultura de la información como un proceso dinámico por medio del cual se logra el mejoramiento de las facultades intelectuales, y el desarrollo de los medios y las formas de comunicación para la generación de conocimiento, comprensión, apropiación y socialización de información cuya finalidad es incrementar el bagaje de conocimientos y experiencias. A la vez se producen avances aplicando la información a la solución de problemas individuales y sociales, y esto genera nueva información.

La cultura de lainformación es causay efecto de haber desarrollado las actitudes y aptitudes para usar la información; y a ella se debe la calidad, cantidad, y disponibilidad de los medios y formas de información y comunicación. A sí, tenemos que a mayor calidad y cantidad de cultura de la información, más se espera avanzar en el proceso (infinito) civilizatorio, lo cual propiciará, a su vez, el crecimiento de la cultura

3 Cf́Ángel Aguirre, Angel. Culturaeidatidadailtural. Introdurciónalaantropdoǵa Barcelona: Bardenas, 1997. p. 17.

4 Carl G. Jung, El honbreysusśnbdos, pp. 89-102.

5 Ángel Aguirre, qp at. pp.7-8. 
informativa. Pero para que ésta pueda convertirse en beneficio social debe estar al servicio de los valore ${ }^{6}$ y derechos universales. ${ }^{7} \mathrm{D}$ elo contrario la civilización puede ponerse al servicio de la agresión y al autoaniquilamiento.$^{8}$

Por lo tanto la sociedad de la información se deberá distinguir por su capacidad para transformar el conocimiento en medios que registren la información con el fin de hacerla accesible a todo aquel que la requiera. En este sentido la tendencia es hacer de la información un patrimonio de la humanidad para que los ciudadanos accedan al recurso que les permitirá "la educación para la vida”. Una reflexión sobre este concepto nos obliga a pensar que el proceso de educación para la vida tiene implicaciones muy profundas, puesto que los ciudadanos deberán estar capacitados para acceder y usar de manera apropiada la información, la cual debe devenir un proceso de aprendizaje, y por lo tanto una transformación que obligará a un nuevo aprendizaje. Se producirá entonces una especie de "banda de Möebius" porque se entrevera información y aprendizaje en un ciclo permanente en el espacio del individuo, y a mayor conocimiento se abrirán nuevas dudas de las que surgirán nuevas necesidades informativas. Así las capacidades de lectura determinarán las posibilidades para informar, informarse y documentar, y por lo tanto esas capacidades deberán superar los obstáculos que la reducen a ser un mero reconocimiento de signos y una actividad banalizada por las ofertas impresas, audiovisuales y digitales que empobrecen los contenidos y a la vez deterioran los procesos cognitivos.

\section{LA CULTURA DE LA INFORMACIÓN EN EL ENTORNO DE LA BIBLIOTECO- LOGÍA}

La cultura de información se expresa en la existencia de la institución bibliotecaria, labibliotecología y las instancias académicas de investigación y formación de especialistas en el campo como parte de la estructura de la cultura de la información. Es a través de todo esto como se ha logrado relacionar el universo de los lectores

6 Cooperación, libertad, felicidad, honestidad, humildad, amor, paz, respecto, responsabilidad, sencillez, tolerancia , unidad.

7 Vida, libertad, igualdad, seguridad, integridad, dignidad, medio ambiente, paz, educación, salud, trabajo, información*, descanso. En: D eclaración Universal de los D erechos Humanos adoptadas y proclamada por laA samblea G eneral de las Naciones Unidas, en la resolución 217ạ(III) de 10 de diciembre de 1948.

* D esantes-Guanter señala "el derecho a la comunicación o su fórmula integrante moderna, el de recho a la información, es así un derecho derivado, que se haido acuñando a través delos siglos. Su grado de evidencia o de proximidad al derecho primario, a la vida, es elevado si se considera al hombre no sólo como ser individual, sino en su dimensión naturalmente, vitalmente, comunitaria." . Cf D esantes-G uanter, José Ma. Univesidady daæehoala doumentaaón Madrid: pp.61-62.

8 Freud, Sigmund. El malestar dela altura. En: O bras completas. Argentina: Amorrotu, 1990 v. 21 (1927-33 ) p.140. 
con el de los documentos mediante procesos de sistematización y organización de los recursos informativos, y su preservación, transferencia, difusión y acceso.

Pero puesto que la cultura de la información es parte del sistema social de comunicación, su participación está determinada por la tradición histórica y la definición de proyectos y políticas a nivel nacional dirigidas a constituir la cultura dela información. Por lo tanto se provocan diferencias entre las naciones y al interior de ellas, situación que se manifiesta en la inversión que se destina para sostener una infraestructura informativa y programas dirigidos a la formación de actitudes, además de aptitudes, necesarias para usar y aprovechar la información en la cual se encuentra involucrada la lectura. Y es precisamente en este terreno en donde se han encontrado deficiencias que limitan a los individuos para ejercer plenamente sus capacidades, acceder alainformación, y saber usarla y aprovecharla en su desarrollo personal yen beneficio del entorno con el que interactúa.

D esde luego a lo anterior hay que añadirle otros factores como el económico y cultural que dificultan el flujo entre individuos y conocimiento. Incluso se puede afirmar que la información es consecuencia o producto del desarrollo y que por tanto se relaciona con el poder económico, científico y tecnológico que diferencia a las potencias de aquellos países donde la tradición de la cultura de lainformación no ha sido atendida y cuyas economías son endebles, lo cual se manifiesta en sistemas bibliotecarios deficientes e insuficientes que afectan la circulación de la información. A esto hay que añadir la pérdiday deterioro del patrimonio documental y bibliográfico, el retroceso o falta de desarrollo de las habilidades de lectura, y las deficiencias y aptitudes para acceder y usar la información, a lo que se suma una pobre infraestructura de tecnología de la información (equipamiento, conexiones, acceso a bases de datos y publicaciones digitales, entre otros), todo lo cual obstaculiza el acceso al conocimiento.

Se esperaría que a mayor cultura de la información más desarrollo. Esto sería posible en la medida en que la información de calidad fuera oportuna, pertinente y disponible, y que los ciudadanos estuvieran preparados para transformar la información en conocimiento que pudiera ser aplicado de manera inmediata o mediata a la actividad humana para resolver problemas y producir nueva información.

A sí cuando se afirma que la información le da poder a quien la posea, debemos matizar que no es el hecho de poseerla lo que otorga el poder sino la capacidad para saber usar lainformación; es en este saber donde radica verdaderamentetal poder.

Ciertamente la información, como ya se mencionó, siempre ha estado presente de manera implícita o explícita en el desarrollo de la humanidad; sin embargo todo indica que hoy el ideal dela sociedad contemporánea, denominada de la información, es que sea una sociedad lectora, informada y documentada. No cabe duda que el arribo de la tecnología de la información y de las telecomunicaciones TIC ha venido a "desvelar" la importancia de la información como recurso estratégico para el desarrollo económico y cultural de las naciones. Ahora el fenómeno informativo se encuentra en una encrucijada, por un lado el derecho a la información: el libre acceso a ella por 
parte de todos los ciudadanos; y por el otro lado la privacidad: los intereses económicos lo han convertido todo en un recurso rentable que se rige por las leyes del mercado en detrimento del bien cultural y social.

Sin duda la cultura informativa ha cobrado enorme visibilidad con la presencia y expansión de Internet, pero no se puede dejar de lado el contexto de esta preocupación que surge de la presión por formar parte de la globalidad. Además la creciente participación de la poderosa industria de la tecnología delainformación y de las telecomunicaciones para impulsar proyectos que favorezcan la innovación, también buscan formas de apoyary dar facilidades para invertir en infraestructura, y para permitir el flujo de la información a lo largo y ancho de nuestro planeta. Pero el exceso de oferta informativaimpresay digital, sumado a problemas de lectura y deficiencias en las aptitudes para acceder y usar esa oferta pueden romper el equilibrio social dada la excesiva oferta de diversas calidades, y la existencia de pueblos con capacidades muy desiguales para acceder, discriminar, usar, y comunicar la información.

La cultura de la información se está fortaleciendo en algunos países con gran tradición; y en otros ha empezado a ganar lugar en las agendas políticas, económicas, y culturales. En algunos, dada su situación: guerra, pobreza, o fundamentalismos, la cultura de lainformación es un tema que aguarda. Pero existeun interés por tratar de "armonizar" al mundo sumando los distintos programas y proyectos que tienen una presencia mundial que avalan los organismos internacionales o regionales (UNESCO ; IFLA; ALA; Unión Europea, etcétera), entre los cuales se encuentran:la preservación del patrimonio bibliográfico y documental del Programa Memoria del Mundo; el control bibliográfico; la transferencia de la información; el acceso a la información; el derecho a la información; los derechos de autor; la formación de aptitudes para accedery usar colecciones impresas, audiovisuales y digitales; la organización del conocimiento; el equipamiento tecnológico, y la biblioteca digital.

Es realmente espectacular la atención que ha cobrado la información y la preparación de la sociedad para acceder a ella y aplicarla para producir desarrollo. D esde 1989, cuando la AmericanLibraryAssociationcreó el Comitee on Information Literacy y el National Forum on Information Literacy ( integrado por representantes de 65 países) han estado trabajando este tema y sus resultados se encuentran plasmados en recomendaciones, normas, estudios y proyectos dirigidos a determinar la formación de ciudadanos aptos para participar en la construcción de la sociedad de la información. Este interés incluso se ha extendido a sectores de profesionales dela bibliotecologíay lainformación en otros países, en los que ya se han formulado declaraciones relacionadas con el desarrollo de habilidades 
informativas o la educación de usuarios con la finalidad de integrarlas en los programes nacionales de educación. ${ }^{9}$

En suma, laTIC, en el contexto de la sociedad de la información y la globalidad, ha propiciado que diferentes sectores, entre ellos los gobiernos, se vean presionados para adoptar los parámetros internacionales con los que se mide actualmente el desarrollo, circunstancia que los hallevado a centrar su atención en laimportancia de la información y las nuevas formas de informar y de informarse. Pero también deben estar pendientes de las innovaciones que generan nuevo conocimiento y atender la capacitación de los ciudadanos para participar en un proceso de aprendizaje permanente que dura toda la vida y produce habilidades que permitan hacer de ella un recurso de desarrollo y de producción de conocimiento.

De tal forma la cultura informativa no se puede reducir al desarrollo de la habilidad para dominar las herramientas computacionales y de telecomunicaciones; el asunto es más complejo e incluye posiciones sociológicas y filosóficas antagónicas, pues para unos la tecnología representa progreso y democratización, en tanto que para otros propicia sometimiento, automatismo, individualismo exclusión o nuevas formas de consumismo que someten cultura alas leyes del mercado impuestas por la industria de la tecnología y los medios masivos de comunicación

Ante esta situación, la institución bibliotecaria tendrá que seguir siendo el factor de equilibrio social al garantizar el libre acceso a la información a todos y la oferta de una lectura de calidad, adecuada y suficiente. Al mismo tiempo habrá de preservar el patrimonio documental y bibliográfico conforme a su precepto fundacional, y gestionar la encomienda de la sociedad moderna en cuanto a compartir la responsabilidad defomentar la lectura y capacitar a la población para que pueda acceder a la información y usarla.

Las instancias bibliotecológicas de casi todos los países atienden ya, en menor o mayor medida, el tema de educación de usuario, ahora denominado alfabetización en información. Incluso se trabaja en acuerdos nacionales, en particular en el sector de la educación superior, dirigidos a la elaboración de programas que desarrollan destrezas para accedery usar la información. A simismo en los últimos años la literatura producida sobre el tema por el sector de bibliotecologíay documentación va en aumento lo que revela que se trata de un aspecto al que se le buscan diferentes alternativas, en particular la forma deintegrarlo al proceso de enseñanzay del trabajo. Sin

9 En México se emitió una Declaratoria entre instituciones de educación superior en el marco del encuentro convocado por bibliotecólogos de la Universidad Autónoma de Ciudad Juárez en octubre de 1997, cuya recomendación fundamental fue la de diseñar e implementar un Programa Nacional de Formación de Usuarios (PRONAF) a fin de que lo hiciera suyo la Red Nacional de Bibliotecas de Educación Superior. Cf Lau Jesús y Jesús Cortés, comp. Desamdlodehabilidadesen insituriones deeduracónsupaior. pp. 129-132. 
embargo el tema de la lectura es aún una asignatura que requiere una atención similar o mayor a la que está recibiendo la alfabetización en información.

\section{LA CULTURA DE LA INFORMACIÓN ¿SIN LECTORES?}

El fenómeno de Internet parece reunir simbólicamente todo lo que entraña ahora la cultura informativa, e incluso es considerado como un corte generacional, es decir una línea entre el antes y el después de Internet, de forma que nos presenta una nueva dualidad en conflicto entre la cultura escrita y la digital; entre quienes pueden y saben usar la tecnología de la información y quienes no, y se habla también de la brecha digital (Digtal Divida de la misma forma que ha sucedido con los pueblos orales en comparación con los letrados, considerados los primeros como arcaicos, silvestres o salvajes 0 , en una palabra, primitivos. Posiblemente dentro de algunos años los grupos que no puedan acceder a la tecnología de la información digital serán agrupados entre los primitivos y escasamente desarrollados o evolucionados. D e tal forma el fenómeno Internet ha venido a poner el énfasis en dos de los temas: la lectura y la educación de usuarios.

La lectura mas que nunca es un asunto de enorme trascendencia para la sociedad de la información, como bien ha señalado S. Murray, "[...]el nivel de alfabetismo (la habilidad para comprender la lectura) es una medida importante de la habilidad de un país para desarrollar una fuerza de trabajo competente con la cual pueda adquirir una ventaja competitiva en la economía global".${ }^{10}$ La afirmación de Murray es cierta pero limitada porque el beneficio no sólo se circunscribe al factor económico, que representa sólo una parte del gran espectro de la civilización.

Todo parece confluir hacia la integración de una sociedad informada e informadora. Y a el D erecho a la Información ha quedado claramente incluido en el artículo 19 de la D eclaración de los D erechos Humanos, y la IFLA creó el Comité de acceso a la información y Libertad de Expresión (CAIFE) durante la Conferencia de 1995 de Estambul. Los principios elaborados por la IFLA están dirigidos a garantizar que:

[...]toda persona debe poder utilizar libre y eficazmente las bibliotecas paraprocurase unainformación permanente, unaindependiente tomade decisiones y el avance en el desarrollo cultural y económico. ${ }^{11}$

Sin embargo resulta paradójico pero se havenido degradando la capacidad de lectura, y por ende el gusto y la práctica cotidiana de leer. En lugar de ello la imagen y la

10 Cf The G lobeand Mail. Scott Murray ( Human Resources D evelopmentCanada- Statistic Canada - Council of Ministers of Education,Canada.) En: Http:/ / www.canadaenespañol.com

11 Cf. Informe preparado para la reunión del Consejo de la IFLA en Copenhague, p.2. 
información oralizada parecen ejerceruna atracción contundente parala mayoría de los habitantes de la naciente sociedad de la información.

En relación con la lectura han surgido problemas graves. En el año 2000, el Programa Internacional de Evaluación de Estudiantes (PISA) de la Organization for Economic Cooperation and Development (OECD) como se señaló, realizó una evaluación en 32 países con el propósito de medir la capacidad para usar los conocimientos (lectura, matemáticas y ciencias) en la solución de retos reales. El estudio se aplicó a 26 mil jóvenes de 15 años que concluyeron la educación obligatoria. En cuanto a la lectura - definida por este organismo como la capacidad de comprender textos, evaluar informaciones, construir hipótesis y aprovechar conocimientos- la finalidad del estudio fue averiguar en qué medida los individuos eran capaces de construir, desarrollar e interpretar el sentido de lo que leen para adquirir y mejorar conocimientos y competencias en otros terrenos.

Como se puede observar en el cuadro que mostramos, laOECD establece tres niveles: la nomalidad establecida por ese organismo se encuentra en un rango descendente 506-494, en el que ese encuentran únicamente cinco países: Noruega, Francia, Estados Unidos, D inamarca y Suiza. D oce países rebasaron esa escala y se encuentran en niveles superiores 545-507; los encabeza Finlandia. En el tercer grupo significativamente se encuentran 14 países por debajo del rango normal, que va del 493 al 396. En este rango están los países que descienden en la escala, aquellos en los que disminuye la capacidad de los jóvenes para localizar fragmentos de información, identificar el tema principal de un texto y establecer una correlación simple con conocimientos corrientes, o limita la lectura en un sentido técnico. Aquí la tendencia es responder con base en lo que creen sin tomar en cuentalo que efectivamente está escrito. No pueden hacer una lectura sofisticada que les permita la detallada comprensión de un texto, ni identificar la relevancia de sus componentes y menos evaluar información de manera crítica y construir hipótesis basándose en la relación de conocimientos que ya poseen con los nuevos que adquieren. Un dato por demás significativo arrojó el estudio es la relación entre género y lectura: las mujeres se desempeñan mucho mejor que los varones en comprensión de la lectura, pero son superadas por sus colegas masculinos en matemáticas y ciencias.

El problema de la deficiencia en las habilidades de lectura representa una grave dificultad que abarca incluso los niveles superiores de educación. En éstos se enseñan, en el mejor delos casos, las razones de los "saberes", pero decía Kant que lo importante no es enseñar razones, sino a razonar. La evaluación menciona el daño infringido por los sistemas pedagógicos que hacen de la lectura un deber ${ }^{12}$ y plantea la

12 "El verbo leer no tolera imperativos. Es una aversión que comparte con algunos otros verbos: amar.. soñar..." Pennac, D aniel. Comounanoda, p. 11. 


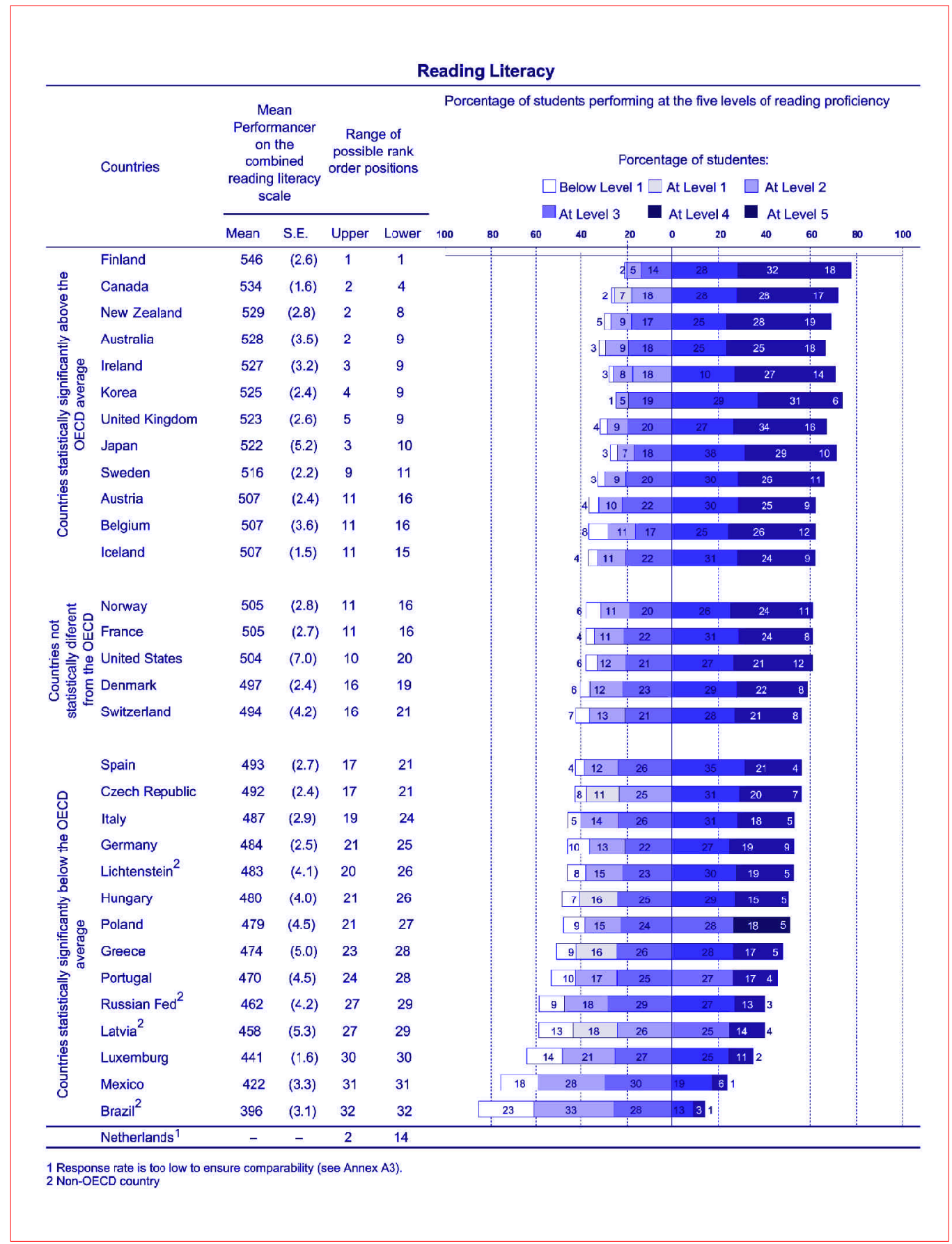

Cf Knowledge and Skills for Life. First Results from PISA 2000. Paris: OECD , 2001. 
oposición ${ }^{13}$ "estudio vesuslectura”. A un cuando no existen investigaciones que avalen esta afirmación, se señala que las instancias educativas han instaurado la lectura como un deber y la han alienado y convertido en una orientación utilitaria con fines inmediatos, con lo cual la despoja de todo placer.

Los factores determinantes que se relacionan con el ámbito de la educación son múltiples, pero destacan tres: la familia, la sociedad y el sistema educativo. D e éstos son la familiay la sociedad los que corren el riesgo de degradarse más fácilmente con los cambios sociales. D onde parecería que deben poner su interés las autoridades es en la ayuda a la enseñanza primaria, que es con toda seguridad en la que pueden compensar eficazmente las deficiencias de las enseñanzas "casera" y "callejera". Lo importante hoy y aquí no es aprender informática o electrónica, es ser capaz de pensar, de razonar sobre ellas. Si no reflexionamos y hacemos algo en relación con lo que nos indica el informe de la OECD, corremos el riesgo de ser una nación de segundo o tercer orden. "La salvación está hoy en aprender a leer y escribir, no sólo a garrapatear y deletrear." 14

Sin duda resulta afortunada la circunstancia actual para sacar alalectura dela opacidad y las inercias en las que quedó atrapada durante la segunda mitad del siglo pasado. D e otra forma la lectura será el principal obstáculo para transitar hacia la sociedad de la información.

Sin duda la evaluación del estudio de la OECD antes citada y que se realizará cada tres años, despertará mayor atención por la lectura y en particular por los procesos pedagógicos en tanto que son responsables de desarticular la lectura del proceso cultural y de reducir la actividad lectora a un mero reconocimiento de signos por privilegiar el aprendizaje memorístico. Sin embargo el problema pedagógico es también consecuencia dela crisis económicas y políticas, además de la historia cultural de los países.

Todo ello se convierte en obstáculos para la lectura, que N. Jitrik,, ${ }^{15}$ sintetiza en tres: uno de naturaleza social y derivado del sistema productivo, que posibilita o dificulta el acceso a los bienes culturales y determina también los ritmos de aculturación, lo cual establece diferencias entre los grupos sociales por lo que toca a la relación con los textos fundamentales, la cual está determinada por cuestiones económicas y la eficacia con la que se puedallevar a cabo la actividad lectora. El segundo obstáculo que propicia un alejamiento de la lectura son los medios masivos de comunicación y su tendencia a la simplicidad, con lo cual magnifican la complejidad de la lectura, e incluso en algunos casos la descalifican y socavan más todavía los intentos de rescatar el gusto por la lectura. El tercer obstáculo radica en el poder con el que se ha investido el acto de leer, basándolo más en creencias que en estudios que

13 Cf Garrido Felipe. Estudiovesuslectura, pp. 43-56.

$14 \mathrm{Http} /$ / etradacomar/ insiturional/ grearal/ frameasp?IdSecion=19 consultado el [31 de enero 2002]

15 Noé Jitrik, Lecturayailtura pp. 14-17. 
fundamenten sus poderes: que la lectura es por sí misma formativa; que leer es sinónimo de comprender conceptos; que hay ciertos textos cuya lectura es obligatoria e importante; que la lectura es necesariamente una actividad gozosa o placentera que todos deben experimentar; etcétera. Todos estos obstáculos se hacen complejos cuando se trata de reducirlos o de transformar su dirección debido al sujeto lector.

Estas ideas o creencias en torno a la lectura no sólo ocultan su carácter productivo sino que limitan el desarrollo, las aptitudes y las actitudes que asumen que la lectura no es una actividad que se da de una vez y para siempre sino una actividad que impplica una práctica constante para que, al igual que cualquier o tra actividad artística o deportiva, llegue a ser productiva y produzca conocimiento y/ o placer.

Así pues, la sociedad de la información tiene una limitación: la falta de lectores con las habilidades necesarias para producir un conocimiento que se constituya en un ciclo de aprendizaje permanente. Se afirma que una de las principales actividades de la alfabetización informativa es la de formar y fortalecer las actitudes y aptitudes lectoras. En suma, la capacidad para hacer de la lectura una actividad productiva es la base para desarrollar la cultura de la información. Pese a que la lectura forma parte del derecho a la educación, los avances en esa materia se reducen, en algunos países, a lo alcanzado por la población alfabetizada, e incluso a la cantidad de libros que se leen, aunque como señala N. Jitrik la idea de que:

[...]a más lectura más cultura, [...] desde una perspectiva alfabetizadora, por ejemplo, esta ecuación es convincente, pero también desde un punto de vista cultural, es insuficiente: por menos que se ahonde en ella se advertirá que, además de numerosa la lectura debe ser rica o elevada o, por lo menos, adecuada. ${ }^{16}$

La tecnología de información y telecomunicación ha empezado a socializarsecon gran rapidez en los últimos diez años y se ha extendido hacia ámbitos distintos al científico y académico hasta instalarse plenamente en la sociedad.

\section{LA NUEVA ALFABETIZACIÓN}

LaAmerican Library Association ha propuesto una definición deInfomationLite ray(alfabetización de la información) que incluye el siguiente conjunto de habilidades para el alfabetizado en información, el cual:

* ha aprendido a aprender,

* sabe cómo se organiza la información,

* es capaz de localizar la información que necesita,

* sabe usar la información de manera que otros puedan aprender de la información que produce. 
En otras palabras José López Y epes ${ }^{17}$ ha definido alos alfabetizados (en información) como individuos capaces de documentar y documentarse en la vida cotidiana; es decir, personas que se han convertido en una especie de homodoumentalis un individuo informado y creador de información que manifiesta su capacidad para seleccionar, encontrar y organizar la información de manera personal para que ésta le sea útil, rentable y aprovechable.

La definición propuesta por la ALA se ha generalizado o globalizado y los diferentes autores y proyectos que abordan el tema aceptan las seis habilidades ${ }^{18}$ propuestas por la ALA, que deben ser desarrolladas por los ciudadanos de la sociedad de la información (algunos añaden el manejo de la computadora), helos aquí:

1. reconocer la necesidad de información;

2. saber formular la pregunta mediante palabras clave, conceptos relevantes y pertinentes y en frases precisas y claras relacionadas con sus necesidades de información;

3. identificar fuentes de información;

4. formular estrategias de búsqueda de acuerdo con las fuentes de información;

5. evaluar la calidad y pertinencia de la información;

6. usar la información; es decir, organizar, analizar, sintetizar, obtener y elegir los datos pertinentes e incorporarlos a su capital de conocimientos, así como aportar nueva información para ser transmitida mediante el ciclo de la comunicación, 0 bien plasmar ese conocimiento en aplicaciones prácticas que le permitan resolver tanto problemas de su vida personal como actividades relacionadas con su formación, su trabajo y sus actividades recreativas.

Estas habilidades para resolver problemas de información están formulabas para poder desempeñarse no sólo en el medio digital presente en los medios de la cultura impresa sino en cualquier otro. D esde luego se incluye aquí el desarrollo de las habilidades para manejar el equipo propio de la tecnología de la información: manejo de los artefactos y del soporte físico para operar computadoras, impresoras, aparatos digitalizadores, modems, tarjetas, teclados, ratones, puertos, grabadoras de CD, conexiones con otros aparatos, configuraciones, programas, lenguajes de marca do ${ }^{19}$ para escribir y comunicar, navegadores, Internet, webs, hipertextos, multimedios. Por otra parte están los medios informativos electrónicos y digitales, como los

17 José López Y epes, Loscaminosdelainfommaón: Cómbluscar, sdecionanyarganizar lasfuentesdenuestra doamentaaión personal. pp-24, 25.

18 Einberg, Micheal B. And Robert Berkowitz. Informationprddemsdving theligsix skillsanaproachtolibraryinformation skillsintrution, pp.5-31.

19 La obra de Ramiro Lafuente López y Roberto G arduño Lengajesdemarcadodedbarmentosdigtales decaráderbibliogáfica constituye un extenso tratado sobre los lenguajes que permiten la construcción de hipertextos que, como señalan los autores , es una nueva forma de interacción con los textos. p 33. 
CD-ROM y las publicaciones digitales: libros revistas, periódicos, además de multimedios, bases de datos, bases de imágenes.

A la vez la comunicación coloquial también ha aprendido el lenguaje propio del medio digital, es el caso del lenguaje icónico y otros códigos visuales que substituyen a la escritura dado que el lenguaje escrito tiene límites. Así, algunas expresiones (alegría, tristeza, etcétera) e incluso sonidos, son llevados a los iconos y a las imágenes, lo cual también implica desarrollar habilidades para su lectura, interpretación y comprensión.

¿Cómo alcanzar la seis aptitudes que recomienda la ALA en una sociedad que lee poco, mal y cuyos contenidos son pobres en lo que a conocimiento se refiere? 0 ¿cómo pueden producirse las habilidades para acceder y usar la información, y además extraer conocimiento y comunicarlo, y más aún, lograruna educación para toda la vida? Por tanto tendríamos que tender a construir una cultura de la información más que reducirnos a alfabetizar para poder manejar la tecnología de la información por medio de la computadora pero con capacidades limitadas para crear conocimiento. Para ello se tendrá que transformar antes el paradigma pedagógico, anclado en la memorización y la repetición, y más bien propiciar el razonamiento pero sobre todo tendríamos que despertar el deseo y el placerpor el conocimiento, campo fértil para desarrollar el gusto por la lectura.

En este proceso la institución bibliotecaria tendría que pasar de un lugar periférico a ser parte del proceso de aprendizaje e informativo permanente, toda vez que es de suponerse que la información y la lectura son los pilares de la educación para la vida. Sobre todo si se pretende que las comunidades académicas lleguen a ser competentes en el acceso y uso de la información ${ }^{20}$ y puedan generary transmitir conocimiento, difícil tarea cuando se observa que la capacidad de lectura de los jóvenes es deficiente incluso en los países desarrollados. Así las cosas no es garantía haber alcanzado un nivel educativo superior o la aptitud para leer textos mecánicamente. Las denominadas "aptitudes" recomendadas por la ALA pasan necesariamente por la lectura crítica y productora de conocimiento y por la capacidad para usar la biblioteca y las fuentes informativas.

\section{CONCLUSIONES}

D e no alcanzar las aptitudes para lograr una lectura crítica y productiva posiblemente no se puedan dominar algunas habilidades para acceder a la información, pero la capacidad para adquirir y mejorar conocimientos y competencias en otros terrenos tendrá serias limitaciones. Es decir: no habrá cultura de la información sin actitudes y aptitudes que hagan de la lectura una actividad productiva de placer y conocimiento.

20 Association of College and Research Libraries. Nomasyaptitudesparad accesoyusodelainfomaiónen la enseñanza supeior. (Aprobadas por la ACRL/ ALA el 18 de enero de 2000) http:/ / www.aab.es 
Y esto sólo se logra si antes se produce el deseo por el conocimiento, aclaro que no únicamente el conocimiento erudito. Sin ese acto donde el lector atraviesa el texto, lo escudriñay encuentra algo que para él tiene sentido, no se da ese encuentro que le permite descubrir algo ignorado o aprender de un modo distinto; es en ese encuentro dondeel lector adquiere el placer de sabery a partir del cual se producirán nuevos deseos. Entonces la lectura se convertirá en un acto gozoso.

\section{O BRAS CONSULTADAS}

Aguirre, Angel. Cultura eidantidad ailtural. Introduccónala antropologá Barcelona: Bardenas, 1997. 273 p.

American Library Association. A progess repat on information literag. An updateon theAmericanLibraryAssociation Presidential Commitee on information literacy. Final report. March 1998. 17 p.

Association of College and Research Libraries. Nomassobreapditudesparad accesoyusodelainformacónenlaensênanzasupeior. Trad. por Cristóbal Pasadas Ureña. Madrid: Universidad Complutense de Madrid, 2001.

Association of College and Research Libraries. Nomassobreaptitudesparad accso y uso dela infomadón en la enseñanza supeior. (Aprobadas por la ACR/ ALA el 18 de enero de 2000) http:/ / www.aab.es

Barthes, Roland. El placerdi textoseguidoporlecióninaugural delacátedradese midoǵa ling̈̈̈́stica de CdlèeedeFrancepronuniada e 7 deenerode1977. 10ª ed. México: Siglo XXI Ed., 2000. 150 p.

Breivik, Patricia S. And E. G ordon G . Informationliterag. Redutionin Library. New York: McMillan Publishing Co., 1989, 250 p.

Dełaracoón Univesal de los Deeehos Humanos adpdadas y prodamadas por la AsambleaGeneal delasNaionesUnidas, enlaresduión217e(III) de10 dedaenbrede1948.

D esantes-G uanter, José Ma. Universidadydarehoaladoumentacoón Madrid: Universidad Complutense de Madrid. D epartamento de Biblioteconomía y D ocumentación, 2001. 135 p.

D ormido Bencomo, Sebastián. « al. Sociedadynuevasteendogáas 3ạed. España: Trotta, 1995. 187 p.

Einberg, Michael B. And Robert Berkowitz, Infomationprodemsdving the bigsix skills AnApproachtolibraryinformationskillsinsturtion, New Y ork: Ablex Publ., 156 p.

Freud, Sigmund. "El malestar de la cultura." En Obrascompleas Argentina: Amorrortu, 1990. Vol. 21. 
Garrido, Felipe. "Estudio versus lectura." Ponencia presentada en la Memoria del Primer Encuentro Internacional sobre Bibliotecas Públicas. Perspectivas en México para el Siglo XXI 2001 México: Conaculta, Dirección G eneral de Bibliotecas, 2001. 265 p. (Biblioteca del Bibliotecario)

IFLA. "Informe preparado para la reunión del Consejo de la IFLA en Copenhague", 6 p. D inamarca: IFLA, 1997.

Jitrik, Noé. Leetrayautura México: UNAM, 1998. 85 p.

Jung, Carl G. El hombreysusśmbdos 6ª ed. España: Caralt, 1997. 334 p.

Lafuente López, Ramiro y Roberto G arduño Vera. Lengrajesdemarcadbde documentosdigitalesdecaráderbibliogáfica México: Centro Universitario de Investigaciones Bibliotecológicas, 2001. 280 p.

Lau,Jesús. Desamdlodehabilidadeseninstituaionesdeeduracoúnsupeior México: Universidad Autónoma de Cd. Juárez, 2000, 134 p.

Lenox, Mary F. and Michael L. Walker "Information literacy : challenge for the Future" En : Intemational Jamal ofInformationandLibraryReserch 4, no 1 (1992) : 1-18.

López Y epes José. Loscaminosdelainfomacớn: cómobuscar, sdeccionaryorganizar lasfuettes denuestra documentaaón pessonal. Madrid: Ed. Fragua, 1997. $345 \mathrm{p}$.

- - -. "La política de la sociedad en España”, Ponencia presentada en las Jomadas deDoumentadónAdministrativay Socięaddela Infomación Zaragoza, 22-24 de octubre de 2001, 33 p.

Luhmann, Niklas, and Raffaele De Georgi. Teenadelasocięad México: U de G; UIA; ITSO, 1993. 444 p.

Murray, Scott. (Human Resarces Dexdopment CanadaStatistics Canada) en: GldbeandMail Council of Ministess of Eduration, Canada, 2001 [cited 24 enero 2002]. Available from http:/ / www.Canada en español

Organisation for Economic Cooperation and D evelopment. Knoweetgeand Skillsfor Life First Results from PISA 2000 Paris: OECD , 2001, 322 p.

Pennac, D aniel. Comounanouda Colombia: Norma, 1997, 168 p.

Ramírez Leyva, Elsa. "Lalectura en la sociedad contemporánea." Investigat dớn Biblidteedógica 15, no. 30 (2001): 114-131.

La Sociedaddela infomaaón: pdítica, teendoǵa eindustria delos conteridos / coord. Mercedes Caridad Sebastián Madrid : Centro de Estudios Ramón Areces ; Universidad carlos III de Madrid, 1999331 p. 